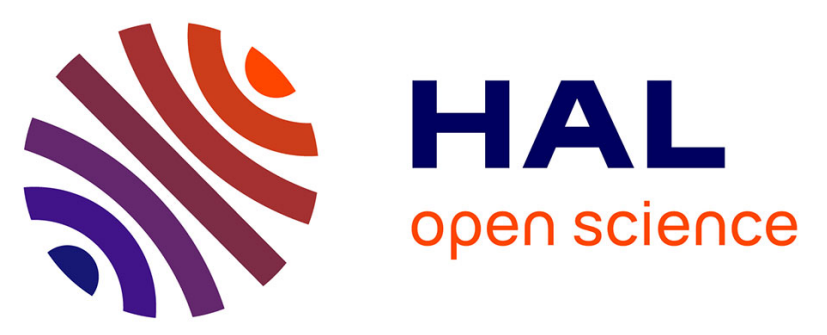

\title{
Time Resolved Observation of the Solvation Dynamics of a Rydberg Excited Molecule Deposited on an Argon Cluster -II: DABCO at Long Time Delays
}

Slim Awali, Jean-Michel Mestdagh, Marc-André Gaveau, Marc Briant, Benoît Soep, Vincent Mazet, Lionel Poisson

\section{To cite this version:}

Slim Awali, Jean-Michel Mestdagh, Marc-André Gaveau, Marc Briant, Benoît Soep, et al.. Time Resolved Observation of the Solvation Dynamics of a Rydberg Excited Molecule Deposited on an Argon Cluster -II: DABCO at Long Time Delays. Journal of Physical Chemistry A, 2021, 125 (20), pp.4341-4351. 10.1021/acs.jpca.1c01942 . hal-03245125

\section{HAL Id: hal-03245125 \\ https://hal.science/hal-03245125}

Submitted on 1 Jun 2021

HAL is a multi-disciplinary open access archive for the deposit and dissemination of scientific research documents, whether they are published or not. The documents may come from teaching and research institutions in France or abroad, or from public or private research centers.
L'archive ouverte pluridisciplinaire HAL, est destinée au dépôt et à la diffusion de documents scientifiques de niveau recherche, publiés ou non, émanant des établissements d'enseignement et de recherche français ou étrangers, des laboratoires publics ou privés. 


\title{
Time Resolved Observation of the Solvation
}

\section{Dynamics of a Rydberg Excited Molecule}

\section{Deposited on an Argon Cluster - II: $\mathrm{DABCO}^{\star}$ at}

\section{Long Time Delays}

Slim Awali ${ }^{\dagger},+, \|$ Jean-Michel Mestdagh, ${ }^{\dagger}$ Marc-André Gaveau,${ }^{\dagger}$ Marc Briant, ${ }^{\dagger}$ Benoît Soep,,$\uparrow$ Vincent Mazet, ${ }^{\S}$ and Lionel Poisson ${ }^{*}, \uparrow$

$\dagger$ †niversité Paris-Saclay, CEA, CNRS, LIDYL, 91191, Gif-sur-Yvette, France.

$\ddagger E M I R$, Institut Préparatoire aux Etudes d'Ingénieurs, Monastir, Tunisie.

๑Université Paris-Saclay, CNRS, Institut des Sciences Moléculaires d'Orsay, 91405, Orsay

§ICube, University of Strasbourg, CNRS, 300 boulevard Sébastien Brant, BP 10413, 67412

Illkirch, France.

||Current address: Équipe Dispositifs Électroniques Organiques et Photovoltä̈que

Moléculaire, LMCN, Faculté des Sciences de Monastir, Université de Monastir, 5019, Monastir, Tunisia.

E-mail: lionel.poisson@universite-paris-saclay.fr

\begin{abstract}
The real-time dynamics of DABCO-argon clusters is investigated in a femtosecond pump-probe experiment where the pump excites DABCO to the $S_{1}$ state within the argon cluster. The probe operates by photoionization and documents the energy and angular distributions of the resulting photoelectrons. The present work complements
\end{abstract}


a former one of our group [Awali et al., Phys. Chem. Chem. Phys., 2014, 16, 516526] where this dynamics was probed at short time, up to 4 ps after the pump pulse. Here, the dynamics is followed up to 500 ps. A multiscale dynamics is observed. It includes a jump between two solvation sites (timescale $0.27 \mathrm{ps}$ ) followed by the relaxation of the solvation cage excess vibrational energy (timescale $14 \mathrm{ps)}$ ) and then by that of DABCO (timescale $>150 \mathrm{ps}$ ). Polarization anisotropy, double polarization and angular anisotropy effects are reported also. They are interpreted (quantitatively for the former effect) in terms of decoherence of rotational alignment, driven by the overall rotation of the DABCO-argon clusters. A tomographic view of the DABCO excited orbital, provided by the double anisotropy effect, is discussed on a qualitative basis.

\section{Introduction}

The 1,4-diazabicyclo-[2,2,2] octane (DABCO) molecule is represented in Fig. 1. Due to the triple bridge between the N-atoms, it has a constrained, fairly spherical geometry. ${ }^{1}$ The electronic structure is quite peculiar, the ground electronic state $\mathrm{S}_{0}$ being of valence character $\left(A_{1}\right.$ symmetry) whereas the first electronic excited state $S_{1}\left(A_{1}\right.$ symmetry, also) has a 90\% 3s Rydberg character. ${ }^{1-7}$ The corresponding single photon excitation is accessible only through a vibronic coupling via the Herzberg-Teller Effect ${ }^{8}$ with $\pi$ bright states. As a result the excitation leads to a slightly polarised orbital, bearing the polarization of the vibronically coupled state. A large expansion of the DABCO electronic cloud is expected upon electronic excitation.

The intrinsic dynamics of DABCO in the $S_{1}$ state has been explored by the present group of authors. ${ }^{4}$ The work was conducted in the real-time domain using the femtosecond (fs) time resolved (TR) photoelectron spectroscopy (PES) technique (fs-TRPES) described by Stolow et al. ${ }^{9,10}$ or Suzuki. ${ }^{11}$ No $S_{1} \rightarrow S_{0}$ relaxation was observed over the 2 ps timescale of the experiment. Instead, an oscillatory behaviour was found and interpreted as a quantum 
interference between two vibrational modes. Rotational decoherence was also observed with a timescale of $360 \pm 100 \mathrm{fs}$. It was attributed to the $\approx 10 \%$ contamination of the excited 3s-Rydberg orbital by non-spherical valence configurations. ${ }^{4}$ The fluorescence lifetime of the $S_{1}$ state was measured in the DABCO vapor to be $1.04 \mu s .{ }^{12}$

When the DABCO molecule is solvated, the expected expansion of the electronic cloud upon electronic excitation likely induces changes in the geometrical arrangement of the species that solvate the molecule. Time dependent solvation is the general process underlaying this effect when the excitation is performed by an ultrashort laser pulse. Its dynamics is driven by couplings between geometrical and electronic degrees of freedom.

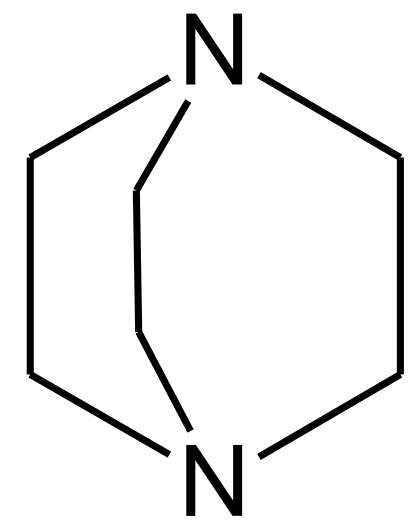

Figure 1: The DABCO molecule.

Time dependent solvation of the DABCO molecule was investigated at short times (0$4 \mathrm{ps}$ ) in a former paper of our group (hereafter referred to as Paper I) ${ }^{13}$ DABCO was bound to the surface of a large argon cluster ( 500 atoms) and was excited to the $\mathrm{S}_{1}$ state by an ultrashort laser pulse. This created a situation where the diameter of the excited orbital is about three times smaller than that of the argon cluster $(\sim 9.5 \AA$ versus $\sim 32 \AA) .{ }^{14}$ A rearrangement of the DABCO-cluster geometry was observed and discussed as a jump between two solvation sites, which differ by the number of argon atoms forming the first solvation shell about DABCO. Both sites are populated in the ground electronic state of DABCO. A substantial site transfer is induced by electronic excitation from the most to 
the least solvating site, i.e. from the site where the largest number of Ar-atoms interact with DABCO to the other site with lesser interacting Ar atoms. These two sites have been unambiguously characterized by their specific photoelectron bands hereafter named track 1 and 2 that exhibit a wavelength dependent intensities.

The present work is built on Paper I. The $\operatorname{DABCO}\left(\mathrm{S}_{1}\right)-\mathrm{Ar}_{n}$ system is investigated again using the fs-TRPES technique but here, a timescale extending up to 500 ps is considered. From Paper I, we do not expect that electronic relaxation or jump between solvation sites could be at play 4 ps after the pump pulse. Instead, we shall see that the 4-500 ps time range is dominated by two relaxation processes, that of the excess vibrational energy and that of the decoherence of the rotational alignment induced by the pump laser. The pump prepares a polarized orbital on DABCO fixed to a rotating ensemble that is probed by a linear polarized ionising laser. The rather slow rotation of the ensemble offers perspective, at least qualitatively, for tomographic information on the excited orbital that is probed.

\section{Methods}

The experimental setup is the same as that used in Paper I and other papers of our group. ${ }^{13-17}$ It is schemed in Fig.2.

Beam generation and pick-up - A cluster source is coupled with a pick-up deposition cell. The beam carrying argon clusters is generated by supersonic expansion through a $100 \mu \mathrm{m}$ nozzle, using a pulse valve (First Sensor Series 9) operating at $20 \mathrm{~Hz}$. With a backing pressure of 16 bar in the nozzle source, the average size of the clusters is estimated to $A r \approx 500 \cdot{ }^{18}$

The cluster beam exits the source chamber through a skimmer and enters into the pickup chamber. A needle is used to fill the latter chamber with a low pressure of DABCO molecules, which are picked-up collisionally by the argon clusters. The pressure in the pickup chamber is maintained low enough so a negligible amount of clusters carry more than one 
DABCO molecule. This was controlled using the CICR method ${ }^{19}$ as explained in Paper I.

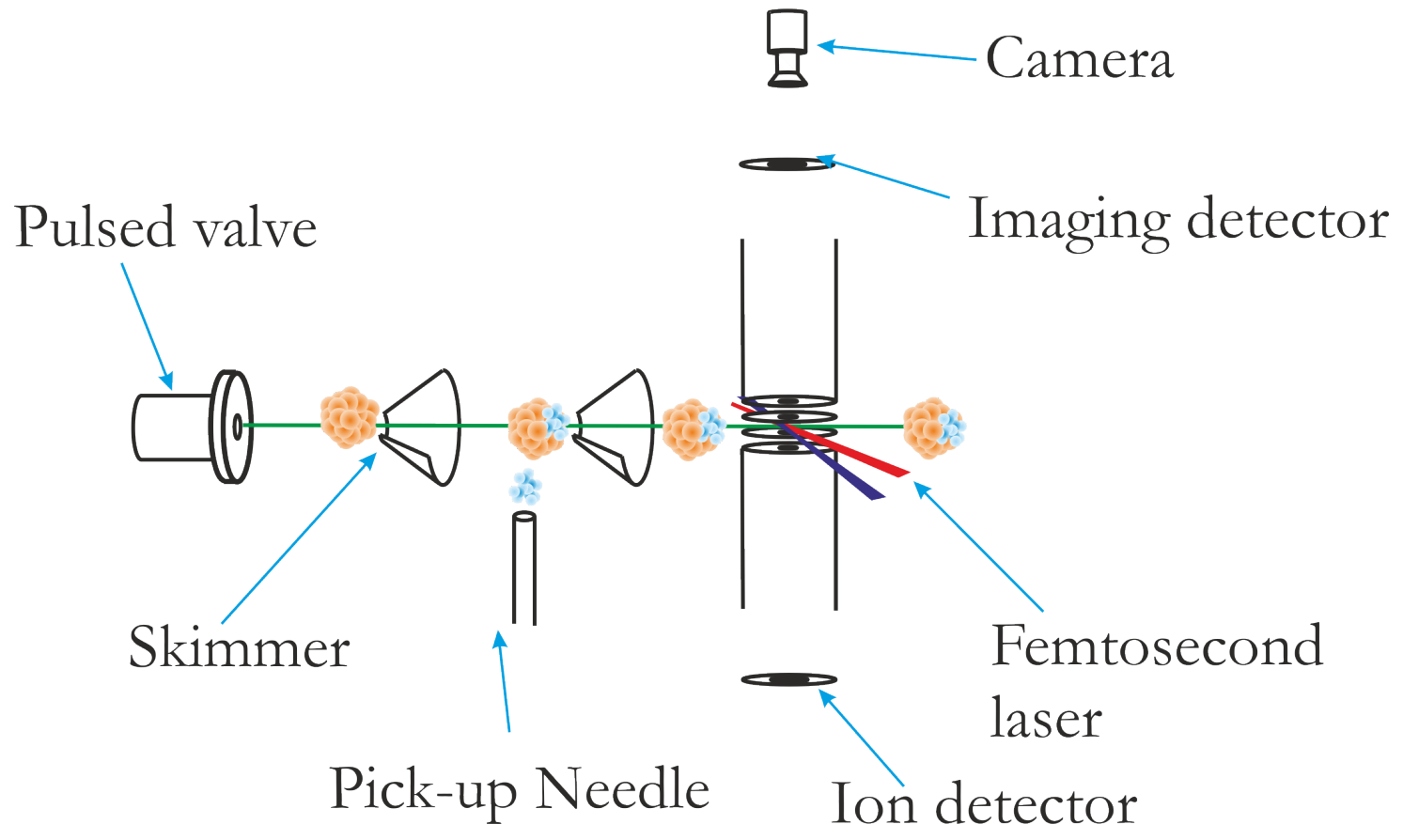

Figure 2: Experimental Setup.

Laser and detection assemblies - The SLIC/LUCA European facility, which operates in the femtosecond regime, is used to pump and probe the DABCO-Ar $\approx 500$ clusters carried by the molecular beam. The operating wavelength of the laser is controlled every day. It varies between 789 and $792 \mathrm{~nm}$ from one day to another. The spectral width (FWHM) is $22 \mathrm{~nm}$. The second and third harmonics of the laser are used here. They are generated by frequency doubling and wave mixing in BBO crystals. The third harmonic (measured within the 265.1-266.3 $\mathrm{nm}$ range) is used to pump the DABCO molecule to the $\mathrm{S}_{1}$ state within the argon environment. The second harmonic $(399-400 \mathrm{~nm})$ probes the electronically excited DABCO-argon clusters by ionization. A delay line built with moving mirrors is used to vary the time delay $\tau$ between the pump and probe pulses. $\tau$ is varied up to 500 ps. The cross-correlation time between the pump and probe laser pulses is measured to $\sim 80 \mathrm{fs}$.

Ions and electrons are extracted orthogonally to the molecular and photon beams. Their velocity and angular distributions are projected on the detector surface of a VMI spectrom- 
eter, where they are monitored. ${ }^{20}$ The extraction and repeller voltages of the VMI need to be switched to detect either photoelectrons or photoions. Only photoelectron detection is reported here.

Importantly, the pump and probe laser beams are linearly polarized. The polarization of the probe laser is parallel to the plane of the Velocity Map Imaging (VMI) detector (labeled Imaging Detector in Figure 2). That of the pump laser is either parallel or perpendicular to this direction.

A full experiment, provides us with two series of raw 2D photoelectron images, one series when the laser polarizations are parallel, the other when the polarizations are perpendicular. The images in each series correspond to a different pump/probe time delay $\tau$.

Image inversion - The radial and angular distributions of the photoelectrons are reconstructed from the raw 2D images by a pBASEX algorithm based on the inverse Abel transform. ${ }^{21,22}$ This treatment decomposes the angular distribution of the photoelectrons into Legendre polynomials, that fits the expected image issued from the projection of a 3D photoelectron distribution having a cylindrical symmetry axis within the projection plan. As seen previously, the collected images can be issued from pump/probe experiments performed with parallel polarization or cross polarization. The decomposition used is also rigorous only for the parallel polarization case. Nevertheless, in both cases we observed similar images without additional contribution. For this reason we applied the same procedure in both case. The inverted images $\mathrm{I}(\mathrm{R}, \theta, \tau)$ appear as the sum

$$
\begin{aligned}
I(R, \theta, \tau)=I_{0}(R, \tau)\left[1+\beta_{2}(\right. & R, \tau) P_{2}(\cos \theta) \\
& \left.+\beta_{4}(R, \tau) P_{4}(\cos \theta)+\ldots\right]
\end{aligned}
$$

where $\mathrm{R}$ and $\theta$ are the polar coordinates of the photoelectrons on the image, $\tau$ the pumpprobe time delay, $\mathrm{P}_{2}$ and $\mathrm{P}_{4}$ the second and fourth order Legendre functions (no odd order appears since the lasers are linearly polarized). After a proper scaling of $\mathrm{R}$ as a 
photoelectron energy $\mathrm{E}$ (see below), a series of spectra $\mathrm{I}_{0}(\mathrm{E}, \tau), \mathrm{I}_{2}(\mathrm{E}, \tau), \mathrm{I}_{4}(\mathrm{E}, \tau)$, ... is obtained. $\mathrm{I}_{0}(\mathrm{E}, \tau)=\mathrm{I}_{0}(\mathrm{R}, \tau) \times \frac{d R}{d E}$ spectra documents the total photoelectron signal, whereas the $\mathrm{I}_{2}(\mathrm{E}, \tau)=\mathrm{I}_{0}(\mathrm{E}, \tau) \times \beta_{2}(\mathrm{E}, \tau)$ and $\mathrm{I}_{4}(\mathrm{E}, \tau)=\mathrm{I}_{0}(\mathrm{E}, \tau) \times \beta_{4}(\mathrm{E}, \tau)$ spectra describe the contribution of photoelectrons distributed as the angular Legendre polynomial of second and fourth order, respectively. The coefficients $\beta_{2}(\mathrm{E}, \tau)$ and $\beta_{4}(\mathrm{E}, \tau)$ are called angular anisotropy parameters. Note that the word angular in "angular anisotropy parameter" is important. Another anisotropy parameter, the "polarization anisotropy parameter" will be introduced later to focus the attention on signal differences whether the polarization directions of the pump and probe lasers are parallel or perpendicular.

The $\mathrm{R}$ (radius in pixel) $\rightarrow \mathrm{E}$ (energy in $\mathrm{eV}$ ) calibration is performed by operating the molecular beam with $\mathrm{O}_{2}$ and photoionizing this molecule as in Paper I. The observed rings in the images are assigned to specific levels of the $\mathrm{O}_{2}{ }^{+}$ion and their radius is fitted to energy for various Repeller voltages (Rep in Volts) and various Extractor/Repeller voltage ratios (Ext/Rep). ${ }^{23}$ The following calibration function is obtained: $\mathrm{E}(\mathrm{eV})=1.52 \times(\mathrm{Ext} / \mathrm{Rep}-$ $0.51) \times(\operatorname{Rep}+77.0) \times \mathrm{R}^{2} \times 10^{-7} \pm 2 \%$.

Track Signal Decomposition (TSD) analysis - The photoelectron bands that characterize the two DABCO cluster types are analysed here as track 1 and 2. Typical raw and inverted photoelectron images are shown in the left and right hand sides of Fig. 3, respectively. Most informative is the inverted image where several broad bands appear. Their evolution as a function of the time delay $\tau$ between the pump and probe pulses is analysed by the Track $\underline{\text { Signal }}$ Decomposition (TSD) method. ${ }^{13,24-26}$ This very specific data treatment was already used in Paper I. It helps extracting information from a series of noisy images where the centre energy and width of the photoelectron bands vary within the series.

It is important to make a distinction between photoelectron bands, which have a well defined significance in physical chemistry, and the numerical tools, which are used to analyse the images. We call tracks the features of the images, which are of physical chemistry 


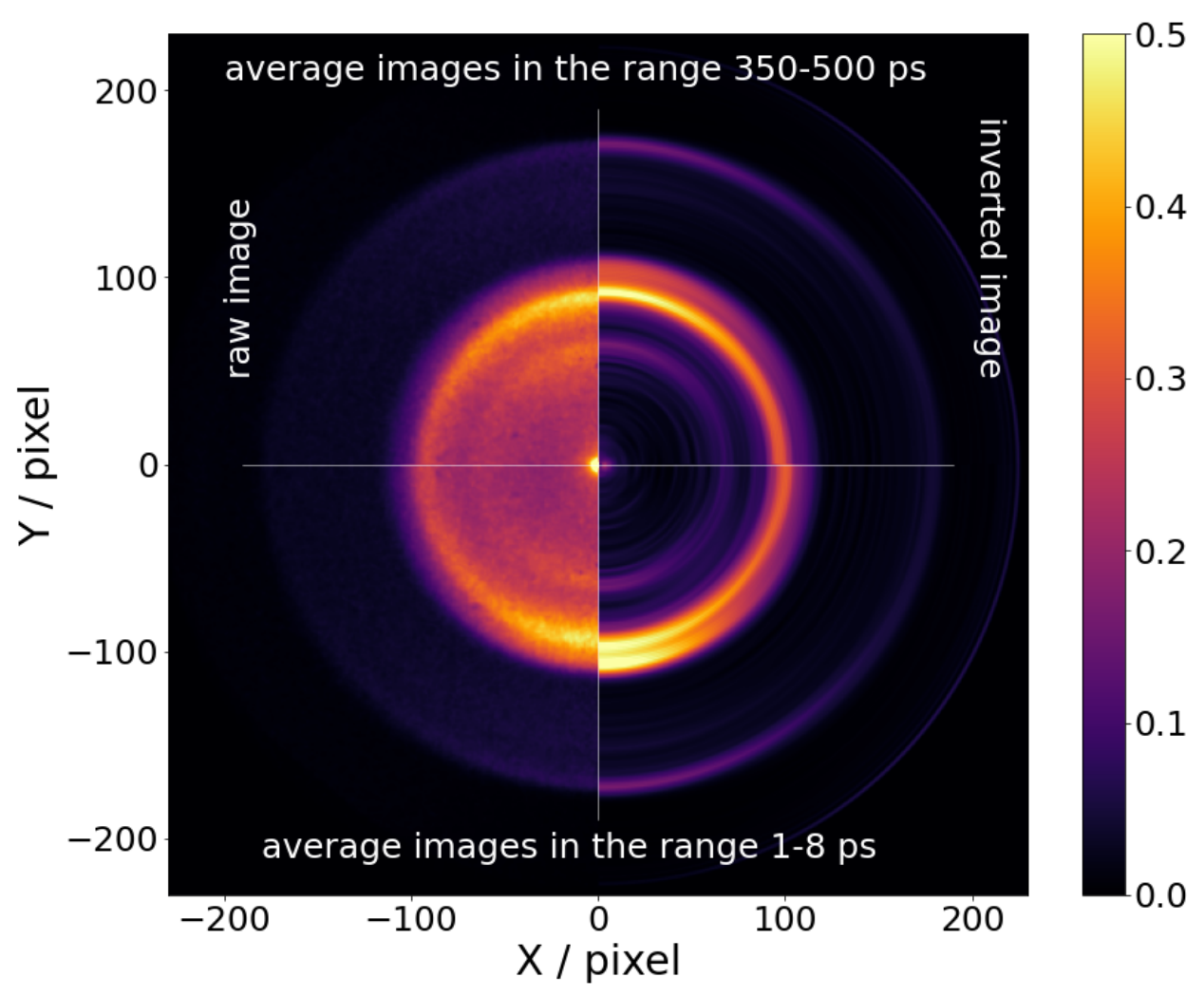

Figure 3: Averaged raw (left side) and inverted (right side) images. In the upper (resp. lower) half of the figure, the average carries on the time delay range 350-500 ps (resp. 1-8 ps).

relevance and need to be followed from one image to another. The tracks are modeled by Gaussian functions which appear as simple numerical tools. Note that in the present work, all the tracks but one are defined by a single Gaussian function. Their centre energy, width and intensity vary as a function of $\tau$.

The TSD method offers a simultaneous decomposition of a whole series of images with a limited number of tracks, assuming constrains to favor a smooth change of the track parameters along the series of images. This provides the time evolution of the track amplitudes, centre energies and full-widths-half-maximum (FWHM). The track intensity is defined as the product of the amplitude by the FWHM.

The TSD treatment of the present spectra follows the same strategy as in Paper I. It is first applied to the series of $\mathrm{I}_{4}$ spectra where tracks are easier to follow. Three tracks are found. The evolution of their centre energy as a function of $\tau$ is used subsequently when 
analysing the $\mathrm{I}_{0}$ and $\mathrm{I}_{2}$ spectra. Then, only the track intensities and widths are adjusted as a function of $\tau$. These widths have not a clear evolution with the pump/probe time delay. They are not reported below.

We already noticed in Paper I that Track 2 is not properly fitted by a single Gaussian function. A better fit is obtained when two Gaussian sub-tracks (Track 2a and 2b) are used with a fixed energy difference of $-0.033 \mathrm{eV}$ between them. Hereafter, the intensity of Track 2 refers to the summed intensities of sub-tracks $2 \mathrm{a}$ and $2 \mathrm{~b}$ and Track 2 energy to that of subtrack 2a only. This is justified because the intensity of sub-track 2a always dominates over that of sub-track 2b. We stated also in Paper I that Tacks 1 and 2 document specifically the dynamics of the DABCO-argon cluster whereas Track 3 reflects that of isolated DABCO molecules, which are also present in the beam.
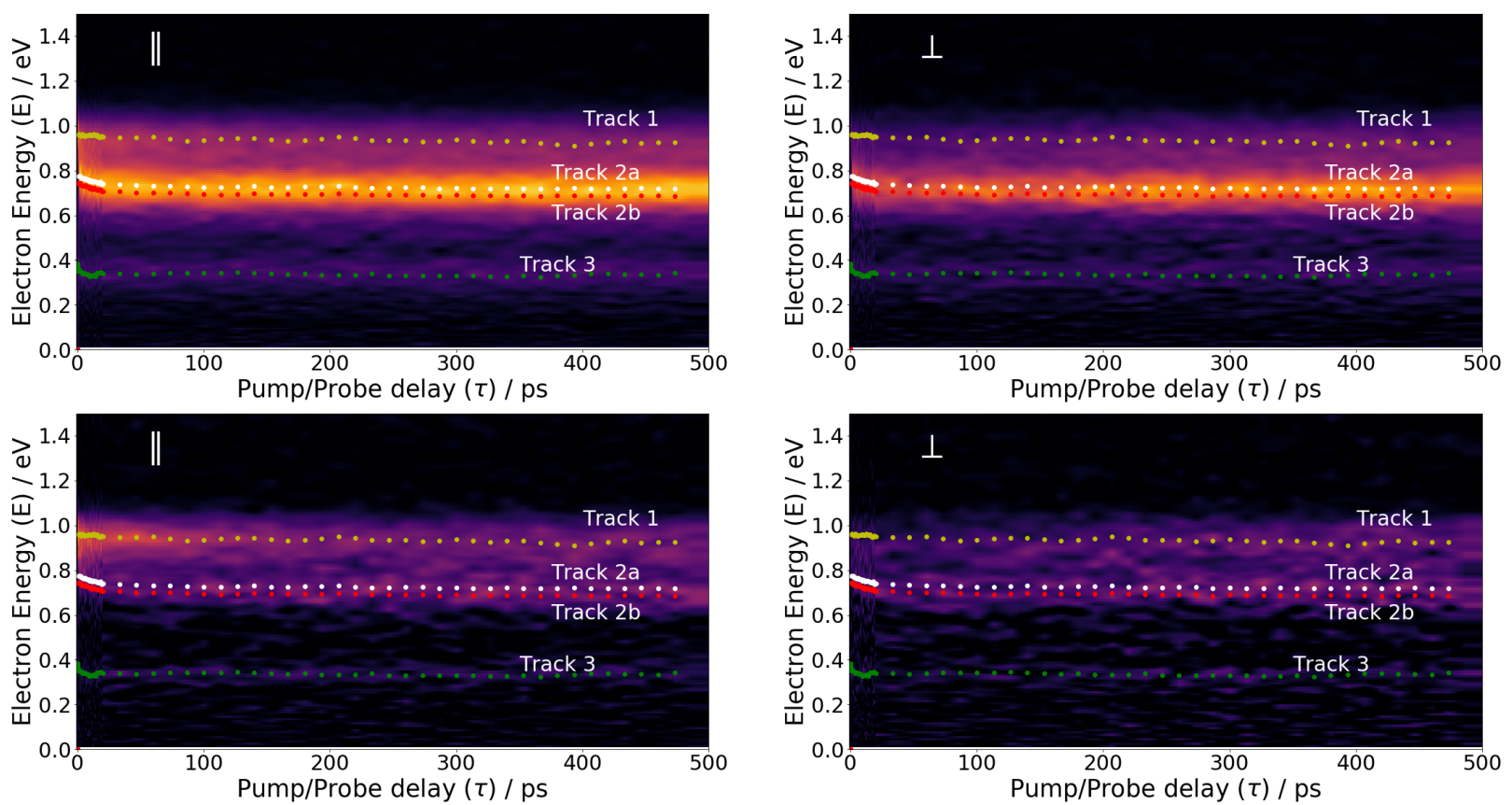

Figure 4: 3D-plot of the time-resolved $\mathrm{I}_{0}(\mathrm{E}, \tau)$ (top panels; total photoelectron signal) and $\mathrm{I}_{2}(\mathrm{E}, \tau)$ (bottom panels; photoelectrons distributed as $\mathrm{P}_{2}$ ) spectra in $265.1 \mathrm{~nm} / 399 \mathrm{~nm}$ pump/probe experiments, $\tau$ being the horizontal scale and $\mathrm{E}$ the vertical one. The laser polarizations are either parallel (left panels) or orthogonal (right panels). The signal intensity is given in false colors using the same color map as in Figure 3. The dots are provided by the track signal decomposition (TSD, see text). 
In summary, the TSD data analysis extracts two quantities from the recorded images: Tracks 1 and 2, which document the dynamics of the DABCO-argon cluster as a function of the pump-probe time delay $\tau$. The tracks are fully characterized by their centre energy $\mathrm{E}(\tau)$ and their intensity $\mathrm{S}(\tau)$. The same centre energy $\mathrm{E}_{\text {Track 1,2 or } 3}(\tau)$ applies, whatever the symmetry of the photoelectron distribution ( $\mathrm{P}_{0}$ or $\mathrm{P}_{2}$ electrons) and the relative polarization ( $\|$ or $\perp$ ) of the lasers. In contrast, $\mathrm{S}_{\text {Track } 1,2 \text { or } 3}(\tau)$ was adjusted for each of theses parameters. The full experimental information is therefore provided by the quantities $\mathrm{E}_{\text {Track 1,2 or } 3}(\tau)$ and $\mathrm{S}_{\text {Track } 1,2 \text { or } 3}^{\| \text {or } \perp, P_{0} \text { or } P_{2}}(\tau)$, given that only tracks 1 and 2 document the desired dynamics of DABCOargon cluster.

\section{Results}

Photoelectron spectra - The $\mathrm{I}_{0}(\tau)$ (top panels) and $\mathrm{I}_{2}(\tau)$ (bottom panels) time-resolved photoelectron spectra are shown in Fig. 4 whether the pump and probe laser polarizations are parallel (left panels) or perpendicular (right panels). The $\mathrm{I}_{0}(\tau)$ spectra document the total photoelectron signal whereas the $\mathrm{I}_{2}(\tau)$ spectra document the contribution of $\mathrm{P}_{2}$ distributed electrons. In these experiments, the pump/probe wavelengths are 265.1/399 $\mathrm{nm}$.

Full experimental information from the TSD treatment - Part of the results provided by the TSD treatment appears as dots in the spectra shown in Fig. 4. They show the centre of the Gaussian peaks which define Tracks 1, 2a, 2b and 3 in the TSD treatment. The quality of the TSD-fit is exemplified in Fig. 5. As said above, tracks 1 and 2 only carry significance regarding the dynamics of the photoexcited DABCO-argon clusters. Their centre energy $\mathrm{E}_{\text {Track 1,2 }}(\tau)$ is shown in Fig. 6 as a function of the pump-probe time delay $\tau$, whereas the signal intensities $S_{\text {Track } 1,2}^{\| \text {or } \perp, P_{0} \text { or } P_{2}}(\tau)$ are reported in Fig. 7. These two figures and the angular anisotropy parameters $\beta_{2 ; \operatorname{Track} 1,2}^{\| \text {or } \perp}(\tau)$ which are reported later in the text (Fig. 11) represent the full experimental information concerning the dynamics of electronically excited DABCO-argon clusters. 


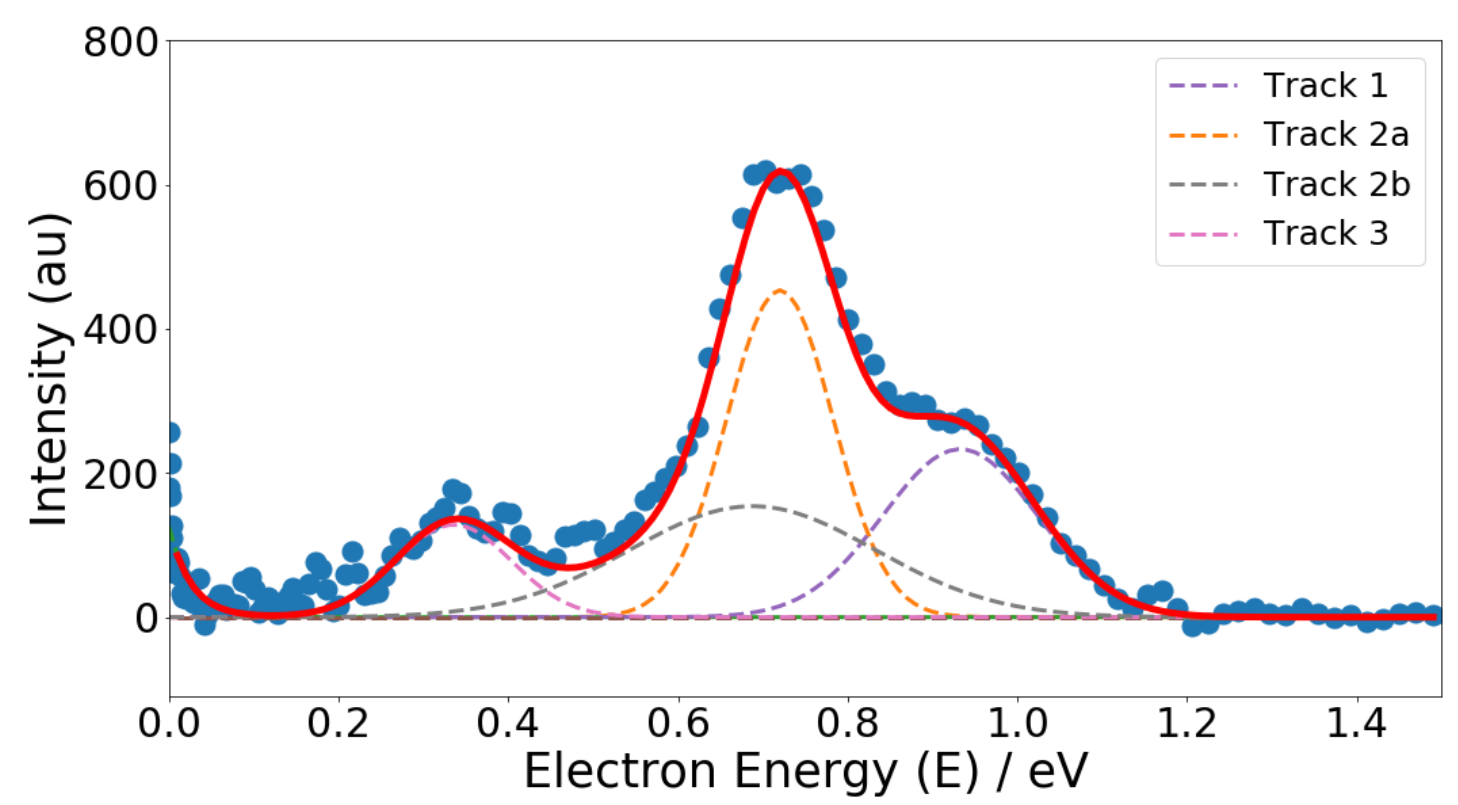

Figure 5: Cut through the top left panel of Fig. 4 at $\tau=433$ ps time delay. The blue dots show the experimental spectrum and the red curve the fit by the TSD treatment. The dashed lines show the contributions of Tracks 1, 2a, $2 \mathrm{~b}$ and 3 .

\section{Discussion}

\section{Intrinsic dynamics of excited $\mathrm{DABCO}\left(\mathrm{Ar}_{n}\right)$ clusters}

The $\frac{S_{T \text { rack } 1,2}^{\|, P_{0} \text { or } P_{2}}(\tau)+2 S_{\text {Track } 1,2}^{\perp, P_{0} \text { or } P_{2}}(\tau)}{3}$ quantities, which appear as dots in the two top panels of Fig. 7 , simulate an isotropic excitation of the $\mathrm{DABCO}\left(\mathrm{Ar}_{n}\right)$ clusters. They reflect the intrinsic dynamics of these clusters after compensation for possible effects due to the alignment of the excited orbital. A slight monotonic increase of the photoelectron signal is observed at the top panel of the figure (total photoelectron signal) for both tracks 1 and 2, whereas a less characteristic situation is observed in the bottom panel of Fig. 7 for the $\mathrm{P}_{2}$ distributed photoelectrons. The signal due to the isolated DABCO molecule appears in Fig. 7 as track 3 (in blue). Since free DABCO has a microsecond fluorescence lifetime, this track is not expected to vary as a function of the time delay. The slight increase that is observed is likely due to a slight misalignment of the delay-line. In the following, we will consider only the photoelectron energies and normalized signal that are not sensitive to this experimental bias. 


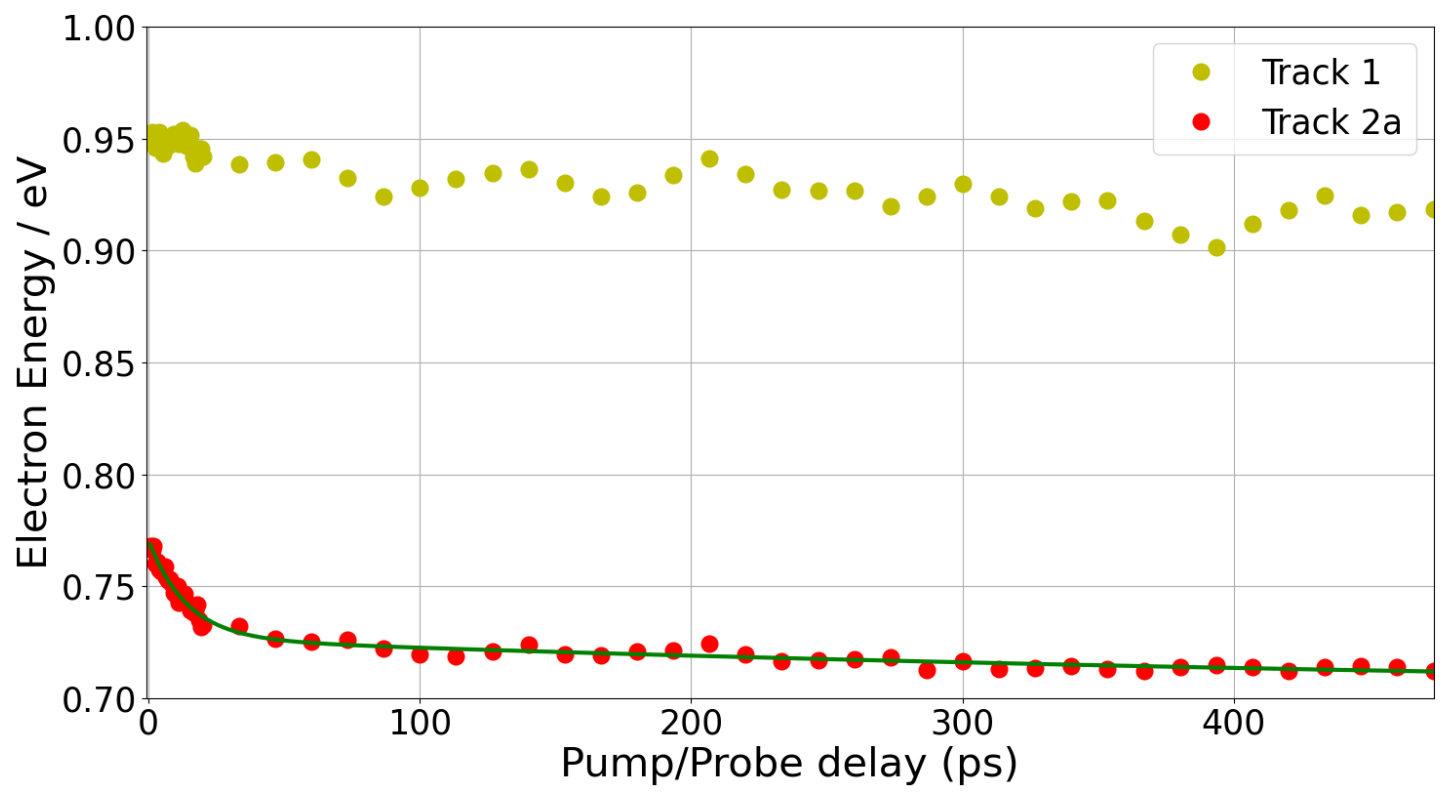

Figure 6: Centre energy $\mathrm{E}_{\text {Track } 1,2 a}(\tau)$ of Tracks 1 and 2a provided by the TSD analysis as a function of the pump/probe time delay $\tau$. The green solid line is a biexponential fit of the track 2 data, using the time constants given in Tab. 1.
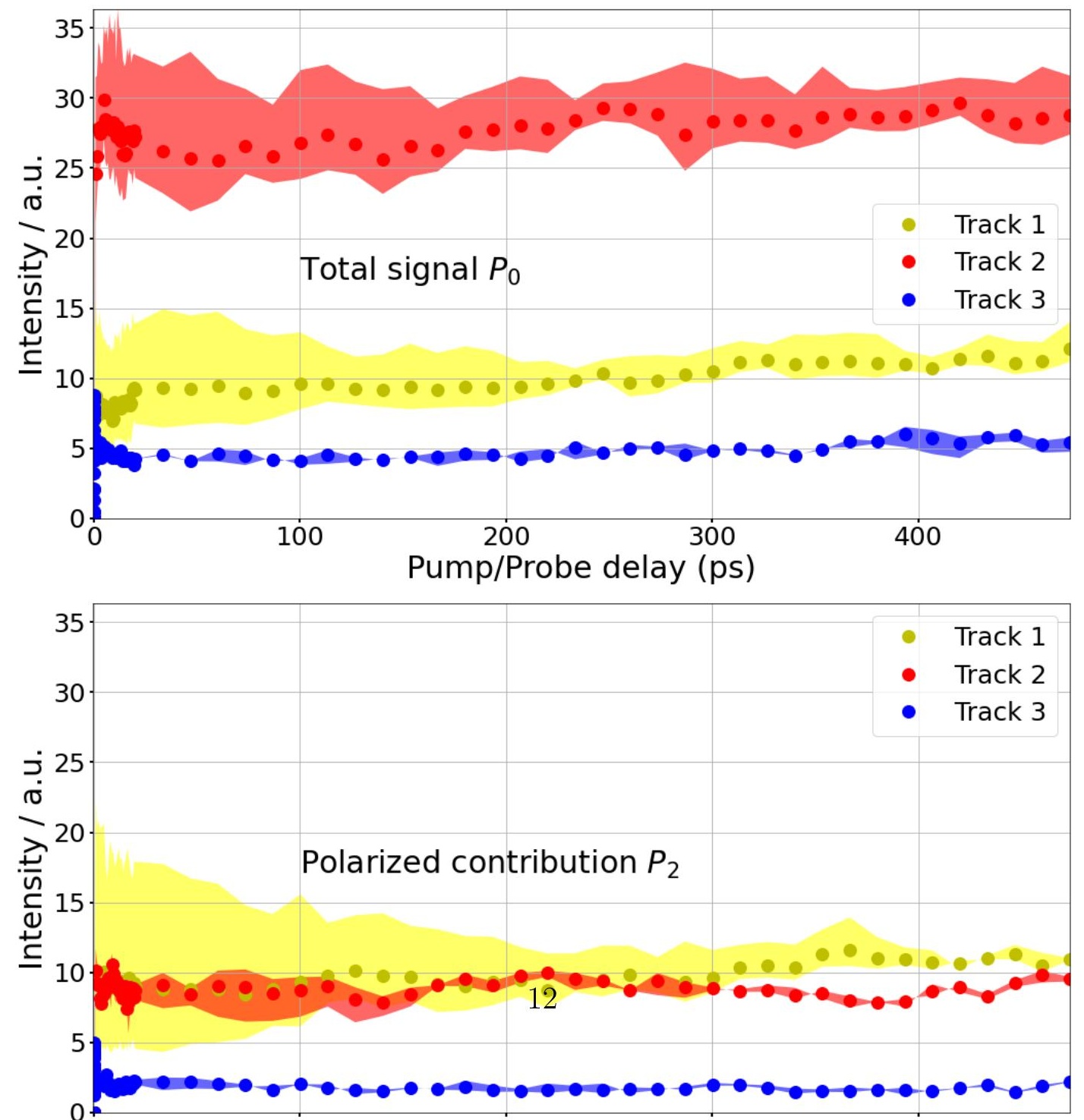
Table 1: Time constants that best fit the centre energies of tracks 1 and 2 shown in Fig.6.

\begin{tabular}{cccccc} 
& \multicolumn{2}{c}{ Centre energy at $\tau=4$ ps } & $\tau_{1}$ & $\tau_{2}$ & $\tau_{3}$ \\
& Paper I & Here & Paper I & Here & Here \\
\hline Track 1 & 0.90 & 0.90 & - & - & $>150$ \\
Track 2 & 0.73 & 0.73 & $0.27 \pm 0.02$ & $14 \pm 1$ & $>150$ \\
\hline
\end{tabular}

The temporal dependence of the centre energy of Tracks 1 and 2 is another information concerning the intrinsic dynamics of the excited $\mathrm{DABCO}\left(\mathrm{Ar}_{n}\right)$ clusters. It is shown in Fig. 6 . The scale of the figure precludes observation of the short time dynamics below 4 ps that is explored in Paper I. The important point here is the behaviour of the Track 1 and 2 centre energies at longer times. The Track 1 signal appears with a monotonic decay that does not reach full relaxation in the time window of the experiment, whereas the Track 2 signal has a bi-exponential behaviour where the slow component does not reach the full relaxation either. A numerical fit of these decay is therefore difficult. It is tentatively reported as time constants $\tau_{2}$ and $\tau_{3}$ in Tab. 1 .

Tab. 1 summarizes the current knowledge of the real-time dynamics of the DABCO molecule deposited on argon cluster after it was excited to the $\mathrm{S}_{1}$ state. From Paper I we know: i) that the DABCO molecule is present at the surface of the argon cluster in two solvation sites, which differ by the number of argon atoms directly bound to DABCO; ii) that Track 1 (resp. Track 2) informs on the most (resp. the least) solvating site; and iii) that the excitation of DABCO to the $\mathrm{S}_{1}$ state turns on an ultrafast dynamics (time constant, $270 \mathrm{fs})$ where DABCO jumps from the most to the least solvating site.

The $\tau_{2}$ and $\tau_{3}$ time constants which appear in the present work likely reflect the time needed for the "hot" DABCO-argon cluster to relax the excess vibrational energy within the argon cluster. A biexponential decay is expected since excess energy stored in vibrational modes of very different nature need to be relaxed: high energy internal modes of DABCO and low energy deformation modes of the DABCO-argon cluster.

The decay times observed for predissociation of Molecule-argon complexes ${ }^{27,28}$ are hardly 
comparable to our measurements. However, the mode-to-mode relaxation time assisted by the van der Waals vibration coordinate as measured on the s-tetrazine-Argon complex ${ }^{29}$ is more relevant. It was measured in the same order of magnitude as time constant $\tau_{3}$, suggesting that $\tau_{3}$ represents the $\mathrm{DABCO}$ to argon bath relaxation time. One can further speculate and assign $\tau_{2}$ to the time constant of local relaxation of the argon bath (see Fig. 8). The latter is turned on by the site-transfer process induced by the sudden enlargement of the DABCO electron cloud upon excitation by the pump laser. The evaporation of argon atoms is believed to be a much slower process with a time-scale in the range of nanoseconds or more ${ }^{30}$ beyond the timescale studied in the present work.

Given the assignment of the $\tau_{2}$ and $\tau_{3}$ time constants, it is interesting to notice that the relaxation process that is associated with $\tau_{2}$ shows up only in the Track 2 data of Fig. 6. This is to be expected since Track 2 documents dynamics associated with the least solvating site. The electronic excitation promotes the transfer of DABCO to this site and only this site (observed through Track 2) has to relax the excess energy due to the site jump. 


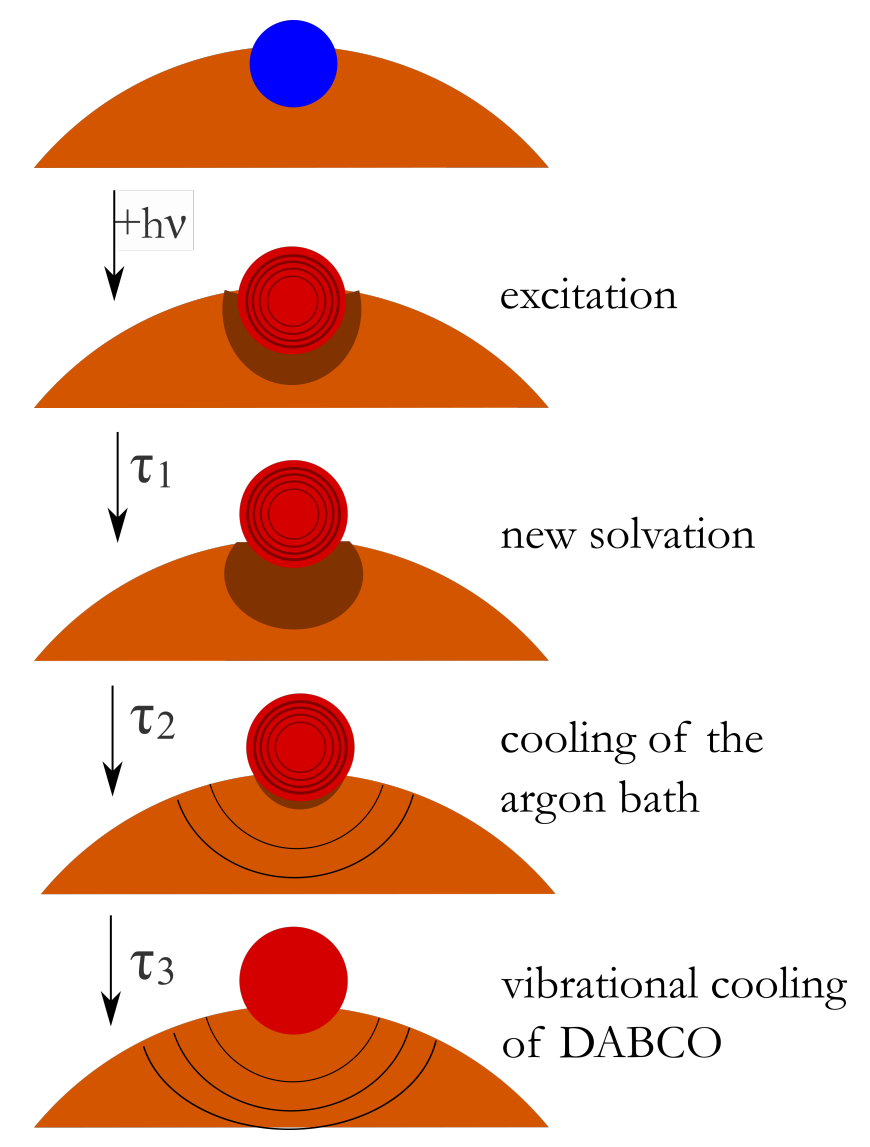

Figure 8: Scheme showing the different timescale of the $\mathrm{DABCO}\left(\mathrm{Ar}_{n}\right)$ photoinduced relaxation. The circular dark lines depict the vibrational excitation.

Mathivon et al. ${ }^{5}$ performed calculations to describe a DABCO molecule in the $\mathrm{S}_{1}$ state, when solvated by 1 to 3 argon atoms. The optimized geometry has the argon atoms in equatorial position with respect to the $\mathrm{N} \cdots \mathrm{N}$ axis, whereas the two polar sites appear as saddle points. When transposing these results to the present context the DABCO molecule may have the $\mathrm{N} \cdots \mathrm{N}$ axis pointing towards the cluster centre, to maximize the number of equatorial Ar-atoms in the most solvating site. Accordingly, three argon atoms would be in equatorial position as in the $\mathrm{DABCO}(\mathrm{Ar})_{3}$ complex, one of the $\mathrm{DABCO} \mathrm{N}$-atom would be free and the other interacting with Ar-atoms in the interior of the cluster. In the least solvating site, DABCO could have the $\mathrm{N} \cdots \mathrm{N}$ axis parallel to the plane of the surface with no argon atom along the $\mathrm{N} \cdots \mathrm{N}$ axis, and only one or two argon atoms would form the first shell. Accordingly the DABCO vibrational energy would be less efficiently coupled to the cluster 
degrees of freedom than in the most solvating site. Hence, the $\tau_{3}$ time constant assigned to this relaxation is expected to be larger for Track 2 (least solvating site) than for Track 1. Unfortunately, the fact that the dynamical process with the $\tau_{3}$ time constant does not reach full completion within the time scale of Fig. 6 precludes a definitive answer on this point.

\section{Polarization anisotropy and rotational coherence}

When comparing the top left and top right panels of Fig. 4 the intensities of tracks 1 and 2 do not have the same behaviour as a function of the pump/probe time delay $\tau$. These differences appear between experiments that differ by the relative polarization direction of the pump and probe lasers (either parallel or perpendicular). They are called polarization anisotropy effects and were documented by Zewail and coworkers from a very general point of view. ${ }^{31-33}$ These authors associated these effects with coherent rotational alignment. We first present their analysis on a qualitative basis. Then, a quantitative description based on numerical simulations is provided. Finally a comparison with experiment is discussed for a further insight into the dynamics of the $\mathrm{DABCO}-\mathrm{Ar}_{n}$ clusters.

\section{A qualitative picture}

Zewail and coworkers considered a population of isotropically distributed molecules (DABCOargon clusters in the present case) and that these molecules are electronically excited via a parallel transition, using a linearly polarized laser of low power density. ${ }^{31-33}$ Among these molecules, the laser excitation selects those whose transition moment is aligned preferentially along the laser polarization. The angular distribution of the excited molecules is thus given by $\cos ^{2} \varphi$ where $\varphi$ is the angle between the transition dipole moment of the molecule and the direction of polarization of the pump laser. This defines the initial alignement of the excited electronic orbital for the ensemble of the rotating systems, i.e. DABCO attached to the argon cluster.

Zewail and coworkers made an important assumption at this point: no intramolecular 
relaxation process scrambles the initial alignment of the excited orbital. In other words, separability is assumed between oscillator strength of the probe transition (whatever the state of the molecule) and intramolecular relaxation if such a process were at play. The effect of this assumption on the present interpretations will be discussed later. With this assumption, the only possible evolution of the initial orbital alignment is a rotation driven by the rotation of molecule (here the DABCO-argon cluster) that carries the electronic excitation.

Of course, molecules in a thermal bath (even a low temperature sample as a molecular beam) do not have all the same angular velocity. The thermal averaging over the molecular rotations causes therefore a gradual loss of the initial coherence between the rotators as a function of time.

This picture is consistent with the decreasing width of the coloured regions in Fig. 7 as a function of time. This justifies the quantitative approach below, which uses the decay of rotational coherences as a guideline for interpreting the experimental data in Fig. 7. Apparently, no rephasing of the DABCO-argon cluster rotation is observed within the time scale of the experiment.

\section{A quantitative treatment}

The polarization anisotropy parameter $r$ - Baskin and Zewail derived general expressions that model the effect of coherent rotational alignement in time-resolved pump/probe experiments. ${ }^{34}$ When transposed to the present context, the analysis by Baskin and Zewail applies to the detection of the isotropically distributed photoelectrons, i.e. the $S^{\| \text {or } \perp, P_{0}}(\tau)$ signals. The polarization anisotropy parameter $r(\tau)$ is a convenient tool to treat of these effects. It is defined as:

$$
r(\tau)=\frac{S^{\|, P_{0}}(\tau)-S^{\perp, P_{0}}(\tau)}{S^{\|, P_{0}}(\tau)+2 S^{\perp, P_{0}}(\tau)} .
$$

Considering that both the pump and the probe lasers operate through parallel electric 
dipolar transitions, Eq. (20) of Baskin and Zewail applies and $r(\tau)$ is given by:

$$
r(\tau)=0.1+0.3 \times \cos 2 \eta(\tau)
$$

where $\eta(\tau)$ is the rotation angle of the DABCO-cluster assembly at time $\tau$ after it has been aligned by the pump laser $(\eta(0)=0)$. The $\eta(\tau)$ quantity does not have the same value according to the size $n$ and rotational state $j$ of the $\mathrm{DABCO}-\mathrm{Ar}_{n}$ clusters. Assuming that the spherical top model applies to the DABCO-Ar ${ }_{n}$ clusters, Eq. 3 can be expanded over $n$ and $j$ :

$$
r(\tau)=0.1+0.3 \times \sum_{n} \sum_{j} \wp_{n} \aleph_{n}(j) \times \cos \left(2 \omega_{n, j} \tau\right)
$$

where $\omega_{n, j}=4 \pi \times B_{n} \sqrt{j(j+1)} / h$ is the angular velocity of an individual DABCO-Ar ${ }_{n}$ cluster in the $\mathrm{j}$ rotational level, $\mathrm{B}_{n}$ being its rotational constant. $\wp_{n}$ in Eq. 4 represents the population of DABCO- $\mathrm{Ar}_{n}$ clusters in the beam and $\aleph_{n}(\mathrm{j})$ the fraction of this population that is in the rotational state $j . \aleph_{n}(\mathrm{j})$ is assumed to follow the Maxwell-Boltzmann statistics at a temperature $\mathrm{T}_{\text {Rot }}$. Given the degeneracy of the rotational levels of a spherical top ${ }^{8}$ is $(2 j+1)^{2}, \aleph_{n}(\mathrm{j})$ is given by:

$$
\aleph_{n}(j)=A \times(2 j+1)^{2} \exp \left[-\frac{B_{n} \times j(j+1)}{k_{B} T_{\text {Rot }}}\right],
$$

where the proportionality factor $A$ insures that $\sum_{j=0}^{\infty} \aleph_{n}(j)=1$.

Since the DABCO-Ar ${ }_{n}$ clusters are generated by pick-up of a DABCO molecule by an argon cluster, $\wp_{n}$ in Eq. 4 is likely proportional to the population of the argon clusters of size $n$ in the beam $\times$ the pick-up cross-section by these clusters. The former quantity is well represented by a log-normal distribution, whereas the second, likely described as the geometrical cross section of the argon cluster, scales as $n^{\frac{2}{3}}$. Note however, that an alternative scaling low has been predicted ${ }^{35}$ and observed ${ }^{36}$ for homonuclear sticking collisions with small 
size clusters. Hence,

$$
\wp_{n}=B \times \frac{n^{\frac{2}{3}}}{n \sqrt{2 \pi}} \exp \left(-\frac{1}{2}\left[\ln (n)-\ln \left(<A r_{n}>e^{-\frac{1}{2}}\right)\right]^{2}\right)
$$

where $<\operatorname{Ar}_{n}>$ is the average number of argon atoms per cluster, prior the DABCO pickup. The standard deviation of the normal distribution, which is associated to the normal distribution, was chosen to be equal to one. With this choice, the FWHM of the log-normal distribution is $<\operatorname{Ar}_{n}>\times \mathrm{e}^{-\frac{1}{2}} \approx 0.6<\operatorname{Ar}_{\mathrm{n}}>$. The proportionality factor $\mathrm{B}$ in Eq. 6 insures that $\int_{0}^{\infty} \wp_{n} d n=1$.

Numerical simulation of $r$ as a function of $\tau$ - Eq. 4 allows simulation of the evolution of the polarization anisotropy parameter $r(\tau)$ as a function of the time delay $\tau$. To achieve that numerically, Eqs. 5 and 6 are injected into Eq. 4 and adequate values must be given to the $B_{n}, \mathrm{~T}_{\text {Rot }}$ and $<\operatorname{Ar}_{\mathrm{n}}>$ quantities.

The rotational constant $\mathrm{B}_{n}$ of $\mathrm{DABCO}\left(\mathrm{Ar}_{n}\right)$ clusters was evaluated as explained in Supporting Information file. The rotational temperature $\mathrm{T}_{\text {Rot }}$ is that of $\mathrm{DABCO}\left(\mathrm{Ar}_{n}\right)$ clusters after the pick-up took place. Supporting Information addresses the effect of transferring angular momentum to the cluster during the pick-up process. It shows that the rotational temperature of the $\mathrm{DABCO}\left(\mathrm{Ar}_{n}\right)$ clusters depends on the size $\mathrm{n}$. An approximate expression $\mathrm{T}_{R o t}=32+\frac{3643}{n^{0.91}}$ is found. It falls in the 40-55 $\mathrm{K}$ range for the $A r_{200-700}$ clusters which play a significant role in the present experiment. 


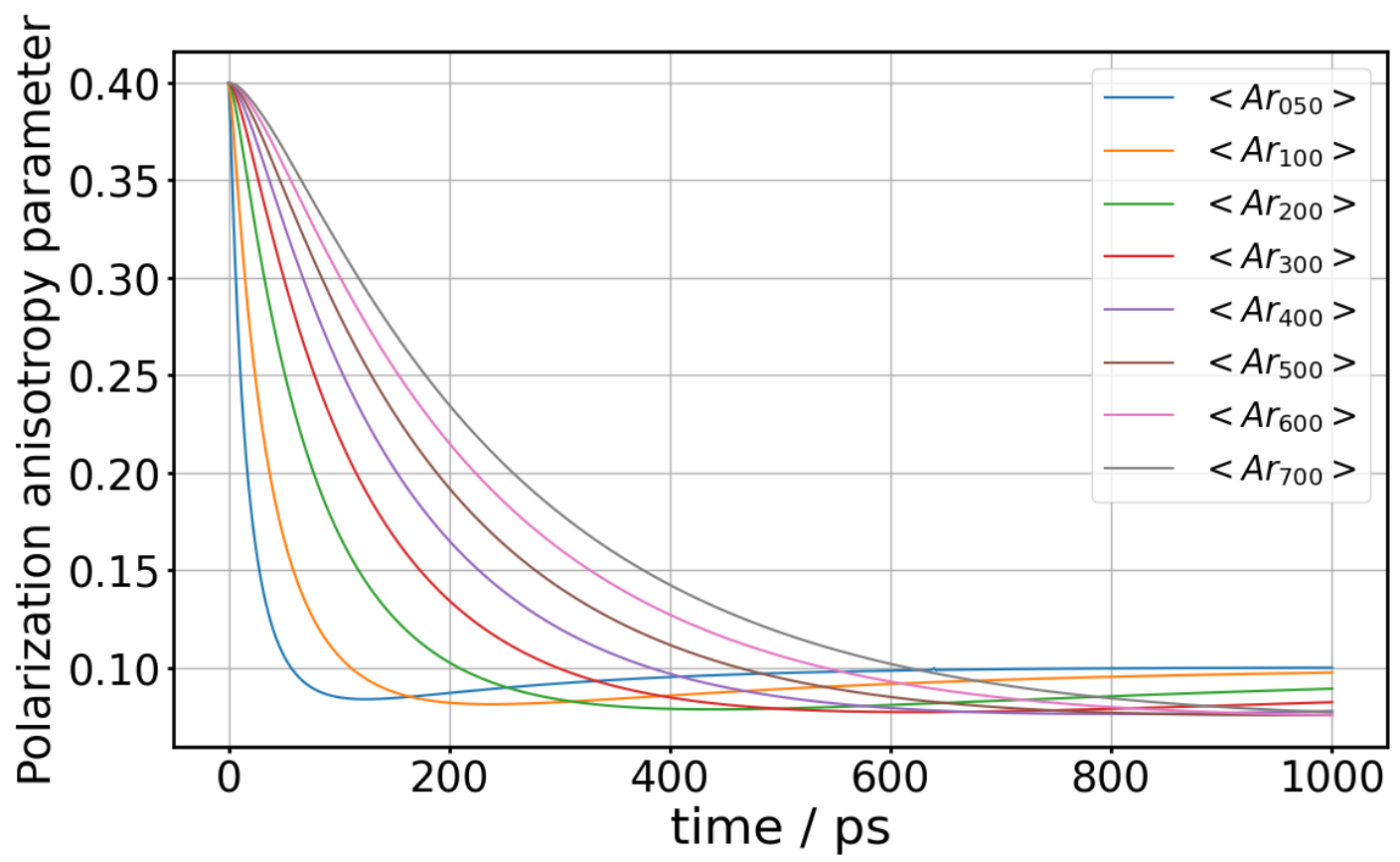

Figure 9: Simulation of the polarization anisotropy parameter $\mathrm{r}$ as a function of the Pump/Probe time delay $\tau$. The curves correspond to a different average size $<\operatorname{Ar}_{n}>$ of the $\mathrm{DABCO}\left(\mathrm{Ar}_{n}\right)$ clusters as labeled in the figure.

Given the values of $\mathrm{T}_{\text {Rot }}, B_{n}$ that have just been discussed, the $\tau$ dependence of the polarization anisotropy parameter $r(\tau)$ was simulated for a series of average cluster sizes ranging between 50 and 700. They are reported in Fig.9. Whatever the cluster size, $r(\tau=$ $0)=0.40$ and $r(\tau)$ decays monotonically towards a plateau within the timescale of the figure. The decay is more rapid and the plateau is higher for the smaller clusters.

\section{Comparison with experiment}

The comparison between the simulations of Fig.9 and the experiment raises a difficulty because of two assumptions made above: i) no intramolecular relaxation process scrambles the initial electronic alignment and ii) both the pump and the probe lasers operate through parallel transitions. This must be clarified before the comparison with experiment is made.

We already recalled from Paper I that the short time dynamics (0-4 ps) of the $\mathrm{DABCO}\left(\mathrm{Ar}_{n}\right)$ clusters is dominated by a switch between two solvation sites, which differ by the number of argon atoms forming the first solvation shell about DABCO. This process has a timescale 
$\tau_{1}=0.27$ ps. In the section above we uncovered that this dynamics is followed by a slower process $\left(\tau_{2}=14 \mathrm{ps}\right.$, see Tab.1) where the excess energy due to the site jump is dissipated within the cluster. We saw also that this dynamics is superimposed on a much slower one $\tau_{3}>150$ ps where the internal energy of the DABCO molecule is dissipated over the cluster.

Among these three processes, the site jump is the more likely to affect the initial alignment of the excited $\mathrm{S}_{1}$ orbital. We discussed indeed that the two sites differ by the position of an argon atom in equatorial position with respect to the $\mathrm{N} \cdots \mathrm{N}$ axis. This modifies the way the excited $\mathrm{S}_{1}$ orbital is further polarized by the argon environment, hence likely affecting the orbital alignement. The effect of the two other processes on the orbital alignment is necessarily more limited. Indeed, they do not affect directly the cluster geometry and are therefore closer to assumption of separability made above between oscillator strength of the probe detection and intracluster relaxation.

Given these considerations, the first few picoseconds that correspond to the site jump process can be ignored when comparing the simulated $r(\tau)$ dependence with the experiment. The initial value $r(\tau=0)$ need simply to be scaled on the experiment. A similar effect has been encountered already and called "dynamic axis switching" by Baskin and Zewail in their work on $\mathrm{HgI}_{2} \cdot{ }^{34}$ These author also scaled simulations to the experiment to account for this effect.

Assumption ii) is considered now. The fact that the excitation by the pump laser is assumed to be a parallel transition causes no difficulty. In contrast, the parallel character of the probe transition is certainly less stringent in the present experiment than assumed in the simulation. The probe operates indeed by ionization and the wavefunction of the escaping photoelectron can accommodate the transition with a non-zero amplitude, whatever the angle between the molecular alignment and the polarization direction of the probe laser. This reduces the absolute value of the polarization anisotropy parameter by a factor that is insensitive to the time delay $\tau$. Hence, scaling the simulations to the experiment as done above with assumption $i$ ) is also the way of relaxing assumption ii). 


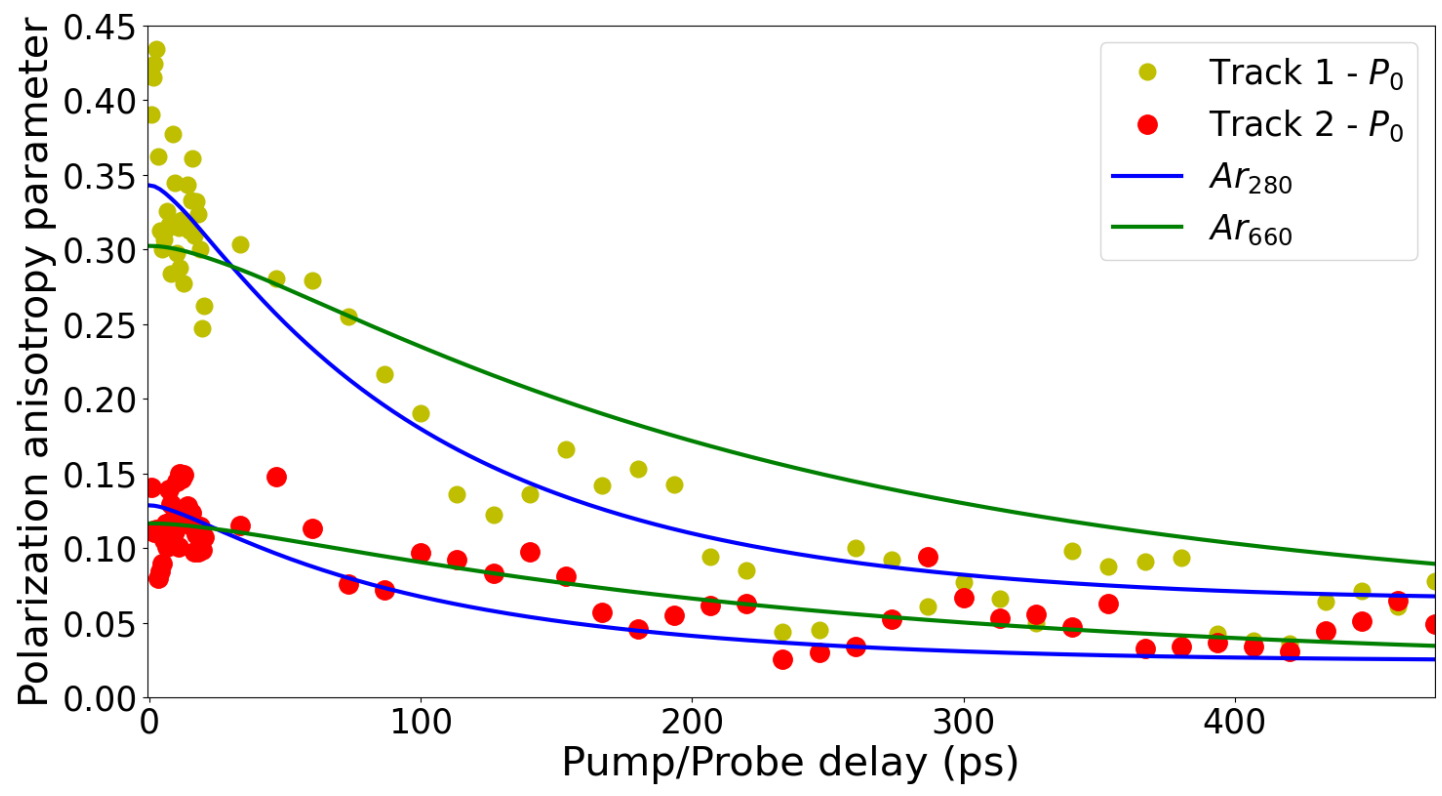

Figure 10: Polarization anisotropy parameter $\mathrm{r}(\tau)$ as a function of the Pump/Probe time delay $\tau$. The experimental results (yellow points for Track 1; red points for Track 2) were calculated from the data in the top panel of Fig. 7. The $\mathrm{r}(\tau)$ parameter simulated for cluster sizes $\left\langle\operatorname{Ar}_{\mathrm{n}}\right\rangle=280$ (blue curves) and $\left\langle\operatorname{Ar}_{\mathrm{n}}\right\rangle=660$ (green curves) were scaled to fit the experimental results (both Track 1 and 2).

Fig.10 reports simulations for $<\operatorname{Ar}_{n}>=280$ and $<\operatorname{Ar}_{n}>=660$, scaled on two sets of experimentally determined $r(\tau)$ values. The later were obtained using Eq. 2 from the experimental data shown in the top panel of Fig. 7. Both simulations go through the experimental points for Track 2, although a better fit is observed with the $<\operatorname{Ar}_{n}>=660$ simulation. In contrast for Track 1, the $\left\langle\operatorname{Ar}_{n}>=280\right.$ simulation much better fits the experimental points than the $<\operatorname{Ar}_{n}>=660$ simulation. A possible interpretation is that the switch from the most (Track 1) to the least (Track 2) solvating site can preferentially occur in the larger clusters. Hence Track 1, when observed at long time delay, documents small clusters where no site jump has occurred. This interpretation is in line with Fig. 1 of the Supporting Information of paper I where the site jump process has no fingerprint on the Track 1 observation. Instead, it shows up as a gradual transformation of Track 2 into track 1 (note that the vertical scale of this figure must be read as "Electron energy" as in the present Fig. 6 instead of "Intensity" as printed erroneously). 


\section{Double polarization and angular anisotropy}

The previous section deals essentially with the polarization anisotropy parameter, built from the total photoelectron signal. The present section still examines polarization anisotropy effects but from the point of view offered by observing the contribution of $P_{2}$ distributed photoelectrons.

As said at the end of Sec. Results, Fig. 11 complements the experimental information provided by Figs. 6 and 7 that are already discussed. The top panel of Fig. 11 shows the angular anisotropy parameters $\beta_{2 ; \operatorname{Track} 1,2}^{\|}(\tau)$ and $\beta_{2 ; \operatorname{Track} 1,2}^{\perp}(\tau)$ as a function of $\tau$. These two parameters differ by the relative polarization of the lasers and do not have the same dependence with the time delay $\tau$. The width of the coloured regions is indeed fairly large at small time delays and becomes smaller as $\tau$ is increased. Hence, double polarization and angular anisotropy effects are observed. A preliminary remark and a few considerations are needed before discussing what additional information is provided by this observation.

Polarization and angular anisotropy have a different origin in the pump/probe process. Polarization anisotropy find its origin in the selection of properly aligned DABCO-argon clusters by the pump laser. It thus carries information on the time evolution of a population created by the pump laser. Angular anisotropy is an information that is created by the probe process from the population created by the pump laser.

To see how this preliminary remark works, we first consider a very simple example: the excitation of a pure p-Rydberg state in an atom in a context where no coupling exists with other electronic states. When choosing the $\mathrm{z}$ axis along the laser polarization, sole the $p_{z}, m_{l}=0$ state is populated. Its population does not change as a function of time both because it is coupled with no other states and because no rotation can mix it with $p_{z}, m_{l} \neq 0$. When probing this state, neither $\beta_{2}^{\|}(\tau)$ nor $\beta_{2}^{\perp}(\tau)$, which can be non-zero because of the polarization of the probe laser, would vary as a function of $\tau$. If a molecule (or cluster) was considered instead of an atom, the initially pure $p_{z}, m_{l}=0$ orbital could be coupled to the molecular rotation and would experience a time dependent mixing with the $p_{x, y}, m_{l}= \pm 1$ 
orbitals. Then, the anisotropy parameters $\beta_{2}^{\|}(\tau)$ and $\beta_{2}^{\perp}(\tau)$ would be $\tau$ dependent.

Finally, still considering a rotating molecule or cluster, if a pure $s$-Rydberg state was excited instead of a p-Rydberg state, the excited orbital would be spherical and would not carry information on the molecular rotation. The $\beta_{2}^{\|}$and $\beta_{2}^{\perp}$ parameters would still be non-zero because the probe laser is linearly polarized but they would be $\tau$ independent. Actually, a small residual $\tau$ dependence would exist because the molecular ion produced by the photoionization probe, may be not spherical and act as fingerprint of the molecular rotation. This was approximately the case in our work on the isolated DABCO molecule where the excited state $\mathrm{S}_{1}$ has a $90 \%$ 3s-Rydberg character. ${ }^{4}$ In this work, the polarization anisotropy parameter $r$, was insufficient to provide a measurable fingerprint of the angular anisotropy parameter $\beta_{2}$.

\section{Characterization of rotational reorientation dynamics and dynamics of the ex-} cited orbital within the argon environment The situation that is met in the present work with the DABCO- $\mathrm{Ar}_{n}$ clusters is different from that was that just discussed for the free DABCO molecule. Here, the angular anisotropy parameters $\beta_{2 ; \text { Track } 1,2}^{\| \text {or } \perp}(\tau)$ are non zero and vary as a function of $\tau$. This tells that the excited orbital that is probed is non-spherical and follows the cluster rotation. Actually, we already know that from section Polarization anisotropy and rotational coherence, but the information here is more direct. It stems from what can be considered as a tomographic investigation of the excited orbital. The latter rotates indeed with respect to the polarization of the probe laser and is therefore probed from different point-of-views when the time delay varies.

We know from our work on the free DABCO molecule that within 90\%, this orbital has a $s$-Rydberg character and is therefore fairly spherical. ${ }^{4}$ The question now is to what extent the tomographic investigation that has just been mentioned can bring information on how the shape of this orbital is changed when DABCO is coupled to the argon cluster.

The $\beta_{2 ; \text { Track } 1}^{\| \text {or }}(\tau)$ parameters, which document Track 1 photoelectrons, are much larger 
and, at least at time delays smaller than 100 ps, they have a different $\tau$ dependence than the $\beta_{2 ; \text { Track } 2}^{\| \text {or }}(\tau)$ parameters, which document Track 2 photoelectrons. This appears in the top panel of Fig. 11. That the $\beta_{2 ; \text { Track } 1}^{\| \text {or } \perp}(\tau)$ parameters are larger than $\beta_{2 ; \operatorname{Track} 2}^{\| \text {or }}(\tau)$ is expected for two reasons that, unfortunately, are difficult to disentangle. First reason: the solvation sites that are probed through these tracks are different. In that case we would conclude that the $s$-Rydberg character of the excited orbital is more distorted when followed through Track 1 (most solvating site) than though Track 2 (least solvating site). Second reason: the population in the least solvating site is partly due to the site jump from the most to the least solvating site, this process being associated with a loss of alignment of the excited orbital.

The latter effect has already been encountered in the present work. The context was slightly different. It was taken into account phenomenologically by: i) scaling the simulated polarization anisotropy parameter on the experimental result and ii) comparing its $\tau$ dependence with experiment. Unfortunately, this cannot be done easily since no theoretical approach is available for modeling the effect of orbital rotation on the $\tau$ dependence of the angular anisotropy parameters $\beta_{2 ; \text { Track } 1}^{\| \text {or } \perp}(\tau)$ and $\beta_{2 ; \text { Track } 2}^{\| \text {or } \perp}(\tau)$. An option could be to transpose to the present context the approach of Baskin and Zewail used above. ${ }^{34}$ As we shall see below, deriving such a rotation-only model without coupling it to the intrinsic dynamics of the DABCO-argon cluster would not be very informative because relaxation dynamics and cluster rotation are difficult to disentangle when double polarization and angular anionotropy is considered. 

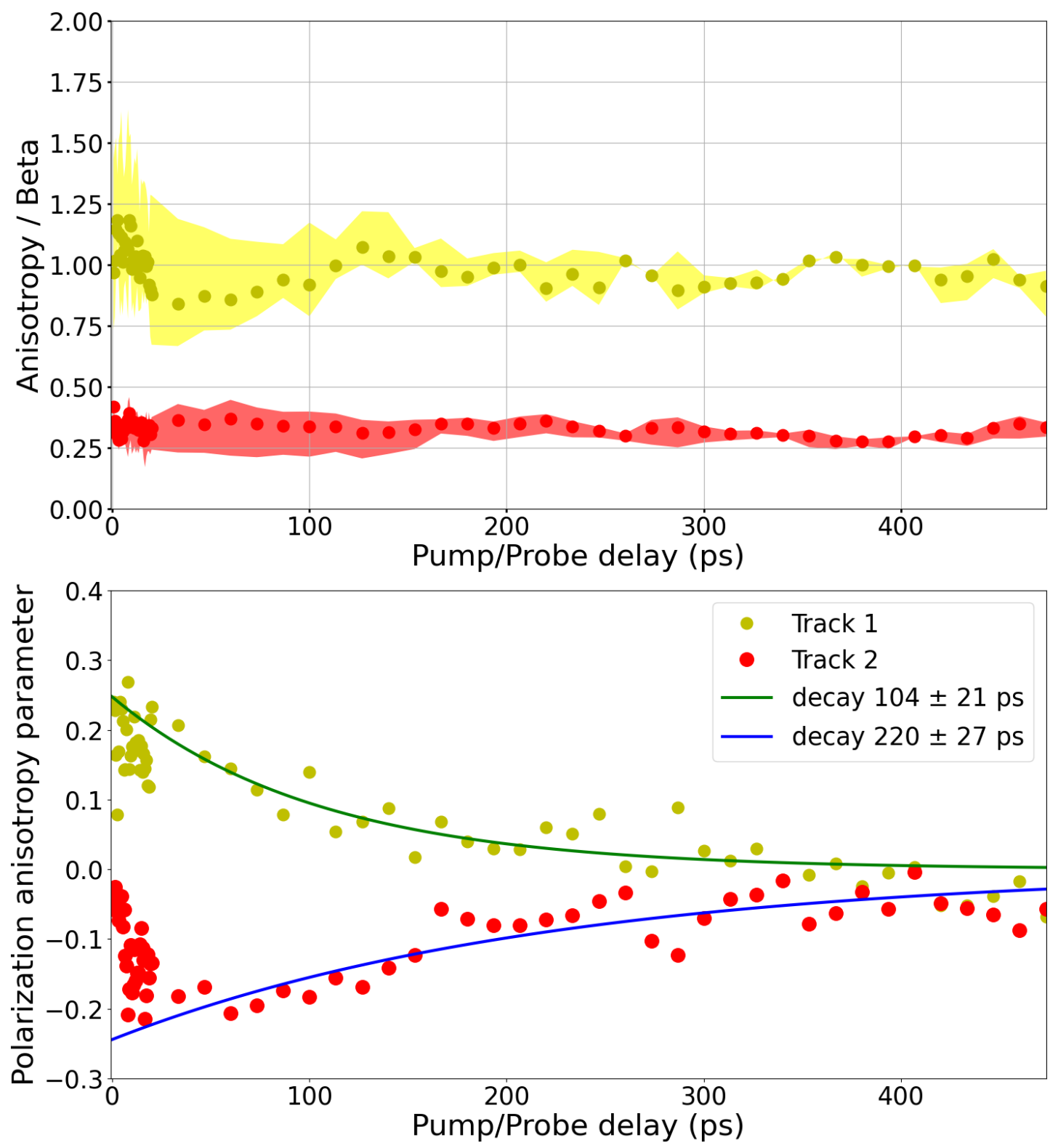

Figure 11: (top) Angular anisotropy parameters $\beta_{2 ; \operatorname{Track} 1,2}^{\| \text {or } \perp}(\tau)=\frac{S_{\text {Track } 1,2}^{\| \text {or } \perp, P_{2}}(\tau)}{S_{\text {Track } 1,2}^{\| \text {or } P_{0}(\tau)}}$ of $\operatorname{tracks} 1$ (yellow) and 2 (red) as a function of the pump-probe time delay $\tau$. The dots show the average values $\frac{\beta_{2 ; \operatorname{Track} 1,2}^{\|}(\tau)+2 \beta_{2 ; \operatorname{Track} 1,2}^{\perp}(\tau)}{3}$. The separation between $\beta_{\text {Track } 1,2}^{\|, P_{0} \text { or } P_{2}}(\tau)$ and $\beta_{\text {Track } 1,2}^{\perp, P_{0} \text { or } P_{2}}(\tau)$ is shadowed in yellow (resp. red) for Track 1 (resp. Track 2). (bottom) Double anisotropy parameter $r_{\beta_{2}, \operatorname{Track} 1,2}(\tau)=\frac{\beta_{2, T \text { Track } 1,2}^{\|}(\tau)-\beta_{2, \operatorname{Track} 1,2}^{\perp}(\tau)}{\beta_{2, \text { Track } 1,2}^{\|}(\tau)+2 \beta_{2, \text { Track } 1,2}^{\perp}(\tau)}$ built from the top panel. $r_{\beta_{2}, \text { Track } 1}(\tau)$ (resp. $r_{\beta_{2}, \text { Track } 2}(\tau)$ is best fitted by an exponential decay (resp. growth) using the time constants given in the insert of the figure.

A parameter of double anisotropy can be defined for Tracks 1 and 2 as $r_{\beta_{2}, \text { Track } 1,2}(\tau)=$ 
$\frac{\beta_{2, \operatorname{Track} 1,2}^{\|}(\tau)-\beta_{2, \operatorname{Track} 1,2}^{\perp}(\tau)}{\beta_{2, \operatorname{Track} 1,2}^{\|}(\tau)+2 \beta_{2, \operatorname{Track} 1,2}^{\perp}(\tau)}$. The corresponding experimental values, deduced from the top panel of Fig. 11, are plotted in the bottom panel of Fig. 11: i) $r_{\beta_{2}, \operatorname{Track} 1}(\tau)$ and $r_{\beta_{2}, \operatorname{Track} 2}(\tau)$ have not the same sign; ii) Track 2 has a peculiar behaviour in the first dozens of picoseconds; iii) both Tracks return exponentially to zero with the time constants given in the insert of the figure.

In the following, we will focus on the effects at long time delays which is driven by the vibrational relaxation, while mechanisms at short times are driven by the site transfer relaxation dynamics. When dealing above with the polarization anisotropy parameter, the situation was favourable since the $\tau$ dependence of $r(\tau)$ was controlled essentially by the cluster rotation. It was discussed indeed that the relaxation dynamics of the excess energy within the cluster and DABCO degrees of freedom has a poor influence on the oscillator strength responsible for the total photoelectron signal. Apparently, a more complex situation is met when double anisotropy parameters $r_{\beta_{2}, \operatorname{Track} 1,2}(\tau)$ are considered: i) unlike $r_{\text {Track } 2}(\tau)$ which could be interpreted with almost rotation-only considerations, $r_{\beta_{2}, \text { Track } 2}(\tau)$ has a complex dynamics that also reflects the relaxation process of $\tau_{2}$ time constant. Cluster rotation and relaxation dynamics seem difficult to disentangle. In contrast ii), the behaviour of $r_{\beta_{2}, \text { Track } 1,2}(\tau)$ at long time delay where $r_{\beta_{2}, 2}(\tau)$ returns to zero more slowly than $r_{\beta_{2}, 1}(\tau)$ seems consistent with the rotation-only effect uncovered with $r_{\text {Track } 1,2}(\tau)$. The clusters probed through Track 2 are indeed larger than those probed through Track 1 and therefore have slower rotation. That, iii) both $r_{\beta_{2}, \operatorname{Track} 1}(\tau)$ and $r_{\beta_{2}, \operatorname{Track} 2}(\tau)$ return to zero at long time delays is the indication that: either the initial alignement of the DABCO molecule on the cluster is fully scrambled as the excess vibrational energy along its internal degrees of freedom is relaxed toward the cluster (process with the time constant $\tau_{3}$ in Tab. 1); or the excited orbital becomes spherical, making the $\beta_{2, \text { Track } 1}^{\|}(\tau)$ (resp. $\beta_{2, \text { Track } 2}^{\|}(\tau)$ parameter progressively equal to $\beta_{2, \operatorname{Track} 1}^{\perp}(\tau)$ (resp. $\beta_{2, \operatorname{Track} 2}^{\perp}(\tau)$ ) and therefore making that the $r_{\beta_{2}, \text { Track } 12}(\tau)$ parameters tend to zero. Again, a situation where cluster rotation and relaxation dynamics are difficult to disentangle is met. This precludes extracting direct tomographic information 
from Fig. 11. A numerical approach that couples rotation effects with the relaxation dynamics with the DABCO argon cluster would be required. This is far beyond the scope of the present work.

\section{Conclusion}

The present paper examines the real-time dynamics of DABCO-argon clusters in a femtosecond pump-probe scheme where the pump pulses excite DABCO to the $\mathrm{S}_{1}$ state within the argon cluster. The probe pulses operate by photoionization and document the energy and angular distributions of the resulting photoelectrons as a function of the pump/probe time delay. The present work complements a former one of our group (Paper I) where this dynamics was probed at short time, up to 4 ps after the pump pulse. Here the dynamics is followed up to $500 \mathrm{ps}$.

When combining the present information with those of Paper I, effects with very different time scales are unraveled. From Paper I we know that the DABCO molecule is excited by the pump laser into the $S_{1}$ electronic state, which has a strong Rydberg character. No state of different electronic configuration is present in a close energetic neighbourhood to promote an ultrafast relaxation of the excess electronic energy through conical intersections. Instead, DABCO stays in the $S_{1}$ state and experiences a jump between two solvation sites with a time constant of $0.27 \mathrm{ps}$. The jump occurs from a most to a least solvating site. These sites are discussed in the present work as related to the alignment of the $\mathrm{N} \cdots \mathrm{N}$ axis of DABCO with respect to the surface of the argon cluster. The present work also reveals that the jump between solvation sites is followed by two sequential relaxation dynamics. One, in the $14 \mathrm{ps}$ regime, corresponds to dissipation within the cluster of the excess vibrational energy due to the site jump, and a very slow one, not completed within the 500 ps of the experiment, where the internal energy of the DABCO molecule is relaxed toward the cluster.

The present work focuses the attention on effects that depends on the relative polarization 
of the pump and probe lasers. This makes further information available. The main one stems from observing the total photoelectron signal provided by the probe laser, whether the linear polarization of the pump laser is set parallel or perpendicular to that of the probe laser. The resulting polarization anisotropy effect is essentially sensitive to the cluster rotation. It appears that the clusters which experience the jump between solvation sties are those of the larger size (clusters of average size 660) within the log-normal cluster distribution (average size 500). Indirectly, this observation reveals that the non-spherical character of the orbital that is probed is enhanced by its interaction with the argon cluster.

Polarization anisotropy also affects the angular distribution of the photoelectrons in the probe process. This double polarization and angular anisotropy offers a tomographic information on the non-sphericity of the orbital that is probed. Because the DABCO-argon clusters rotate in the laboratory reference frame, the angle between the alignment of the excited orbital and the polarization direction of the probe laser changes as a function of the time delay between the pump and probe laser pulses. Hence, the parameter of double polarization and angular anisotropy varies as a function of the time delay. This variation carries information on the shape of the orbital that is probed.

Unfortunately, the corresponding tomographic information remains qualitative because the effects of cluster rotation and internal energy relaxation within the cluster degrees of freedom cannot be disentangled. Both, affect the double anisotropy parameter and their dependence with the time delay occurs with similar time scales. Nevertheless, we hope that the present observations pave the way for future theoretical developments where both the tomographic and multiscale dynamical effects are combined. In our view, that might be done by treating the rotation analytically in the same spirit as done here for the polarization anisotropy parameter and the dynamics numerically in the spirit of what was done by Calvo et al. on the fragmentation dynamics of rare gas cluster. ${ }^{37}$ 


\section{Acknowledgement}

L.P. thanks Dr. Osman Atabek for fruitfull discussions. S.A. thanks EU-ITN project ICONIC-238671 for funding. L.P. thanks the ANR for support through the contract ANR09-JCJC-0090-01 "CHROMADYNE". Furthermore, we thank the CEA/SLIC staff, in particular Olivier Gobert and Michel Perdrix, for technical support.

\section{Supporting Information Available}

Evaluation of the rotational constants $\mathrm{B}_{n}$ of the clusters and estimation of the rotational temperature of the clusters after a sticking pick-up.

\section{References}

(1) Shang, Q. Y.; Bernstein, E. R. Energetics, Dynamics, and Reactions of Rydberg State Molecules in Van-Der-Waals Clusters. Chem. Rev. 1994, 94, 2015-2025.

(2) Halpern, A. M.; Roebber, J. L.; Weiss, K. Electronic Structure of Cage Amines: Absorption Spectra of Triethylenediamine and Quinuclidine. J. Chem. Phys. 1968, 49, $1348-1357$.

(3) Parker, D. H.; Avouris, P. Multiphoton Ionization Spectra of Two Caged Amines. Chem. Phys. Lett. 1978, 53, 515-520.

(4) Poisson, L.; Maksimenska, R.; Soep, B.; Mestdagh, J. M.; Parker, D. H.; Nsangou, M.; Hochlaf, M. Unusual Quantum Interference in the S-1 State of Dabco and Observation of Intramolecular Vibrational Redistribution. J. Phys. Chem. A 2010, 114, 3313-3319.

(5) Mathivon, K.; Linguerri, R.; Hochlaf, M. Systematic Theoretical Studies of the Interaction of 1,4-Diazabicyclo 2.2.2 Octane (Dabco) with Rare Gases. J. Chem. Phys. 2013, $139,164306$. 
(6) Shang, Q. Y.; Moreno, P. O.; Dion, C.; Bernstein, E. R. The Effect of NonpolarSolvents on Rydberg States - Vanderwaals Complexes of Azabicyclooctanes. J. Chem. Phys. 1993, 98, 6769-6778.

(7) Shang, Q. Y.; Moreno, P. O.; Li, S.; Bernstein, E. R. Solvation Effects on the Molecular 3s Rydberg State - Azab/Cyclo Octanes Clustered with Argon. J. Chem. Phys. 1993, $98,1876-1887$.

(8) Bernath, P. F. Spectra of Atoms and Molecules; Oxford University Press: New-York, 2005.

(9) Stolow, A.; Bragg, A. E.; Neumark, D. M. Femtosecond Time-Resolved Photoelectron Spectroscopy. Chem. Rev. 2004, 104, 1719-1757.

(10) Stolow, A. Femtosecond Time-Resolved Photoelectron Spectroscopy of Polyatomic Molecules. Annu. Rev. Phys. Chem. 2003, 54, 89-119.

(11) Suzuki, T. Femtosecond Time-Resolved Photoelectron Imaging. Annu. Rev. Phys. Chem. 2006, 57, 555-592.

(12) Halpern, A. M. The Vapor State Emission from a Saturated Amine. Chem. Phys. Lett. 1970, 6, 296-298.

(13) Awali, S.; Poisson, L.; Soep, B.; Gaveau, M.-A.; Briant, M.; Pothier, C.; Mestdagh, J.M.; Rhouma, M. B. E. H.; Hochlaf, M.; Mazet, V.; Faisan, S. Time Resolved Observation of the Solvation Dynamics of a Rydberg Excited Molecule Deposited on an Argon Cluster-I: Dabco* at Short Times. Phys. Chem. Chem. Phys. 2014, 16, 516.

(14) Lietard, A.; Piani, G.; Briant, M.; Gaveau, M.-A.; Faisan, S.; Mazet, V.; Soep, B.; Mestdagh, J.-M.; Poisson, L. Self-Trapping Relaxation Decay Investigated by TimeResolved Photoelectron Spectroscopy. Phys. Chem. Chem. Phys. 2018, 20, 1120611214. 
(15) Poisson, L.; Raffael, K. D.; Soep, B.; Mestdagh, J. M.; Buntinx, G. Gas-Phase Dynamics of Spiropyran and Spirooxazine Molecules. J. Am. Chem. Soc. 2006, 128, 3169-3178.

(16) Poisson, L.; Raffael, K. D.; Gaveau, M. A.; Soep, B.; Mestdagh, J. M.; Caillat, J.; Taieb, R.; Maquet, A. Low Field Laser Ionization of Argon Clusters: The Remarkable Fragmentation Dynamics of Doubly Ionized Clusters. Phys. Rev. Lett. 2007, 99, 103401.

(17) Poisson, L.; Roubin, P.; Coussan, S.; Soep, B.; Mestdagh, J. M. Ultrafast Dynamics of Acetylacetone (2,4-Pentanedione) in the S2 State. J. Am. Chem. Soc. 2008, 130, 2974-2983.

(18) Awali, S.; Gaveau, M. A.; Briant, M.; Mestdagh, J. M.; Soep, B.; Gobert, O.; Maksimenka, R.; Poisson, L. Multipronged Mapping to the Dynamics of a Barium Atom Deposited on Argon Clusters. Phys. Chem. Chem. Phys. 2016, 18, 32378 - 32386.

(19) Mestdagh, J. M.; Gaveau, M. A.; Gee, C.; Sublemontier, O.; Visticot, J. P. Cluster Isolated Chemical Reactions. Int. Rev. Phys. Chem. 1997, 16, 215-247.

(20) Eppink, A.; Parker, D. H. Velocity Map Imaging of Ions and Electrons Using Electrostatic Lenses: Application in Photoelectron and Photofragment Ion Imaging of Molecular Oxygen. Rev. Sci. Instrum. 1997, 68, 3477-3484.

(21) Garcia, G. A.; Nahon, L.; Powis, I. Two-Dimensional Charged Particle Image Inversion Using a Polar Basis Function Expansion. Rev. Sci. Instrum. 2004, 75, 4989-4996.

(22) Poisson, L. Lv_Pbasex. 2009.

(23) Baklanov, A. V.; Janssen, L. M. C.; Parker, D. H.; Poisson, L.; Soep, B.; Mestdagh, J.M.; Gobert, O. Direct Mapping of Recoil in the Ion-Pair Dissociation of Molecular Oxygen by a Femtosecond Depletion Method. J. Chem. Phys. 2008, 129, 214306.

(24) Mazet, V. Joint Bayesian Decomposition of a Spectroscopic Signal Sequence. Signal Processing Letters, IEEE 2011, 18, 181-184. 
(25) Mazet, V.; Faisan, S.; Masson, A.; Gaveau, M. A.; Poisson, L.; Mestdagh, J. M. Joint Bayesian Decomposition of a Spectroscopic Signal Sequence with Rjmcmc. IEEE Workshop on Statistical Signal Processing 2012, Ann Arbor, USA, 253-256.

(26) Mazet, V.; Faisan, S.; Awali, S.; Gaveau, M.-A.; Poisson, L. Unsupervised Joint Decomposition of a Spectroscopic Signal Sequence. Signal Processing 2015, 109, 193-205.

(27) Osborn, D. L.; Alfano, J. C.; Vandantzig, N.; Levy, D. H. Vibrational Predissociation and Intramolecular Vibrational-Relaxation in Dimethyl-S-Tetrazine-Argon Complexes. J. Chem. Phys. 1992, 97, 2276-2283.

(28) Nimlos, M. R.; Young, M. A.; Bernstein, E. R.; Kelley, D. F. Vibrational Dynamics of Aniline(Ar)1 and Aniline(Ch4)1 Clusters. J. Chem. Phys. 1989, 91, 5268-5277.

(29) Heppener, M.; Kunst, A. G. M.; Bebelaar, D.; Rettschnick, R. P. H. Time Resolved Mode-to-Mode Vibrational-Energy Flow in the Isolated S-Tetrazine Argon Complex. J. Chem. Phys. 1985, 83, 5341-5342.

(30) Calvo, F.; Parneix, P. Accurate Modeling of Sequential Decay in Clusters over Long Time Scales: Insights from Phase Space Theory. J. Chem. Phys. 2007, 126, 034309.

(31) Felker, P. M.; Zewail, A. H. Purely Rotational Coherence Effect and Time-Resolved Sub-Doppler Spectroscopy of Large Molecules. I. Theoretical. J. Chem. Phys. 1987, $86,2460-2482$.

(32) Baskin, J. S.; Felker, P. M.; Zewail, A. H. Purely Rotational Coherence Effect and TimeResolved Sub-Doppler Spectroscopy of Large Molecules. Ii. Experimental. J. Chem. Phys. 1987, 86, 2483-2499.

(33) Felker, P. M.; Zewail, A. H. In Jet Spectroscopy and Molecular Dynamics; Hollas, J. M., Phillips, D., Eds.; Springer Netherlands: Dordrecht, 1995; pp 181-221. 
(34) Baskin, J. S.; Zewail, A. H. Femtosecond Real-Time Probing of Reactions .15. TimeDependent Coherent Alignment. J. Phys. Chem. 1994, 98, 3337-3351.

(35) Vigué, J.; Labastie, P.; Calvo, F. Evidence for N1/3 Dependence of the Sticking CrossSection of Atoms on Small and Medium-Size Van Der Waals Clusters. Eur. Phys. J. D 2000, 8, 265-272.

(36) Lengyel, J.; Pysanenko, A.; Poterya, V.; Slavíček, P.; Fárník, M.; Kočišek, J.; Fedor, J. Irregular Shapes of Water Clusters Generated in Supersonic Expansions. Phys. Rev. Lett. 2014, 112, 113401.

(37) Calvo, F.; Bonhommeau, D.; Parneix, P. Multiscale Dynamics of Cluster Fragmentation. Phys. Rev. Lett. 2007, 99, 083401. 


\section{Graphical TOC Entry}

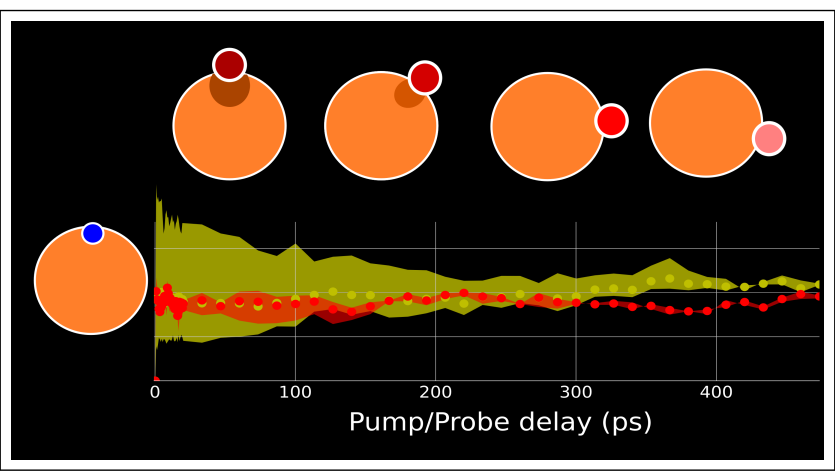

